\title{
Subject Biomarker Domain
}

National Cancer Institute

\section{Source}

National Cancer Institute. Subject Biomarker Domain. NCI Thesaurus. Code C106571.

A special purpose domain that associates the subject with the medical conclusions

contained in the PB domain based on the observations reported in PF. 